\title{
REFERENCES
}

1. R. A. Moore and Z. Nehari, Nonoscillation theorems for a class of nonlinear differential equations, Trans. Amer. Math. Soc. 93 (1959), 30-52.

2. F. V. Atkinson, On second order nonlinear oscillations, Pacific J. Math. 5 (1955), 643-647.

3. W. F. Trench, On the asymptotic behavior of solutions of second order linear differential equations, Proc. Amer. Math. Soc. 14 (1963), 12-14.

4. I. T. Kiguradze, On the asymptotic behavior of solutions of the equation $u^{\prime \prime}+a(t) u^{n}=0$, Math. Reviews 27 (1964), no. 386.

5. B. Viswanatham, A generalization of Bellman's lemma, Proc. Amer. Math. Soc. 14 (1963), 15-18.

6. E. Hille, Nonoscillation theorems, Trans. Amer. Math. Soc. 64 (1948), 234-252.

Sandia Laboratory, Albuquerque, New Mexico

\section{THE DOMAIN OF UNIVALENCE OF CERTAIN CLASSES OF MEROMORPHIC FUNCTIONS ${ }^{1}$}

\section{RAYMOND J. DISTLER}

1. Introduction. Let $K$ be a closed set of points in the complex plane and let $\mathcal{F}(K)$ be the family of functions

$$
f(z)=\sum_{k=1}^{n} \frac{A_{k}}{z-a_{k}}, \quad A_{k}>0, \quad k=1,2, \cdots, n,
$$

where all of the poles lie in $K$. In this paper we shall find the domain of univalence for the class $\mathcal{F}(K)$. By this we mean a domain $U=U(K)$ such that each $f(z) \in \mathcal{F}(K)$ is univalent in $U$, but if any open set is adjoined to $U$, then there is an $f_{0}(z) \in \mathcal{F}(K)$ that is not univalent in the enlarged domain.

In this direction Cakalov [2], [3] has proved two beautiful theorems.

THEOREM A. If all of the poles of (1) lie in the circle $|z| \leqq 1$, then $f(z)$ is univalent in the domain $|z|>\sqrt{ }(2)$, and this domain is maximal for the class of all such functions.

CoRollary. Let $\alpha(t), t \in[-\pi, \pi]$, be nondecreasing, with at least one point where it is increasing. Then

$$
f(z)=\int_{-\pi}^{\pi} \frac{d \alpha(t)}{z-e^{i t}}
$$

Presented to the Society, January 24, 1964; received by the editors July 5, 1963.

1 This paper is part of the author's dissertation done at the University of Kentucky. 
is univalent in $|z|>\sqrt{ }(2)$, and this domain is maximal for the class of all such functions.

THEOREM B. If all of the poles of (1) lie in the interval $-1 \leqq z \leqq 1$ then $f(z)$ is univalent in $|z|>1$ and this domain is maximal for the class of all such functions.

CoROLlary. Let $\alpha(t), t \in[-1,1]$ be nondecreasing with at least one point where it is increasing. Then

$$
f(z)=\int_{-1}^{1} \frac{d \alpha(t)}{z-t}
$$

is univalent in $|z|>1$ and this domain is maximal for the class of all such functions.

We will prove a similar type theorem, valid for each closed point set $K$. This theorem contains Čakalov's two theorems as special cases, and indeed our proof of the main theorem (Theorem 1) is at least as simple as either of the proofs of Theorems A and B.

2. The main theorem. We recall $[1$, p. 213] that a director curve for a conic is by definition the set of all points $P$ such that the conic subtends an angle $\pi / 2$ from the point $P$. It is well known that if the conic is an ellipse the director curve is a circle (see $\$ 3$ below) and that for a parabola the director curve is a straight line. We extend this definition as follows:

Definition 1. Let $K$ be a closed set of points. The director set of $K$ is the collection $D$ of all points $P$ such that $K$ subtends an angle $\pi / 2$ at $P$.

It is clear that the director set of $K$ is the same as the director set of the smallest convex set that contains $K$ (called the convex hull). Further if $K$ is a bounded set, the boundary of this convex cover is a simple closed curve, and consequently the director set $D$ is also a simple closed curve. It is easy to show that if $K$ is bounded then $D$ is star-like with respect to any point in $K$, but as the example at the end of this section shows $D$ need not be convex.

If $K$ is unbounded, then $D$ may be empty, or it may be a simple curve extending to infinity. In any event, whenever $D$ is a simple curve it will divide the plane into two domains, one of which will contain $K$, and the other, which we denote by $U=U(K)$, will consist of all points at which $K$ subtends an angle less than $\pi / 2$.

THEOREM 1. Let $K$ be a closed set for which $U(K)$ is not empty. Then $U(K)$ is the domain of univalence of the class of functions $\mathcal{F}(K)$, i.e., the functions of the form (1). 
Proof. Let $z_{1}$ and $z_{2}$ be distinct points of $U$. Then

$$
\frac{f\left(z_{1}\right)-f\left(z_{2}\right)}{z_{2}-z_{1}}=\sum_{k=1}^{n} \frac{A_{k}}{\left(z_{1}-a_{k}\right)\left(z_{2}-a_{k}\right)} .
$$

Since the set $\left\{a_{k}\right\}$ subtends an angle less than $\pi / 2$ at $z_{1}$ there is a $\theta_{1}$ such that

$$
\theta_{1}-\pi / 4<\arg \left(z_{1}-a_{k}\right)<\theta_{1}+\pi / 4
$$

for each $a_{k}$. Similarly there is a $\theta_{2}$ such that

$$
\theta_{2}-\pi / 4<\arg \left(z_{2}-a_{k}\right)<\theta_{2}+\pi / 4
$$

for each $a_{k}$. From (5) and (6) it is clear that the points $\left(z_{1}-a_{k}\right)\left(z_{2}-a_{k}\right)$ lie in the half plane $\theta_{1}+\theta_{2}-\pi / 2<\arg w<\theta_{1}+\theta_{2}+\pi / 2$. The points $1 /\left(z_{1}-a_{k}\right)\left(z_{2}-a_{k}\right)$ are interior points of a related half plane, and since $A_{k}>0$ for all $k$, the sum in (4) is not zero [4, p. 1]. Thus $f\left(z_{1}\right) \neq f\left(z_{2}\right)$.

To show that this domain of univalence cannot be enlarged, let $z_{1}$ be any point on the director curve $D$. Then there are points $a_{1}$ and $a_{2}$ in $K$ such that $z_{1}-a_{1}$ is perpendicular to $z_{1}-a_{2}$. Let

$$
\begin{aligned}
& \lambda=\left|z_{1}-a_{1}\right|>0 \\
& \delta=\left|z_{1}-a_{2}\right|>0
\end{aligned}
$$

and set

$$
f_{0}(z)=\frac{\lambda^{2}}{z-a_{1}}+\frac{\delta^{2}}{z-a_{2}} .
$$

Then $f_{0}(z)$ is in $\mathcal{F}(K)$ and $f_{0}^{\prime}\left(z_{1}\right)=-\lambda^{2} /\left(z-a_{1}\right)^{2}-\delta^{2} /\left(z-a_{2}\right)^{2}$. Clearly $f_{0}^{\prime}\left(z_{1}\right)=0$ for the two terms each have unit modulus and differ in argument by $\pi$. Therefore if the domain of univalence $U$, which has $D$ as a boundary, is enlarged by the addition of any open set to form a domain $U^{*}$, there is a function $f_{0}(z)$ in our class that is not univalent in $U^{*}$.

Since $U(K)$ is unchanged when $K$ is replaced by the convex hull of $K$, we may assume at the outset that $K$ is convex without loss of generality.

We can replace the sum in (1) by a Stieltjes integral as follows.

Theorem 2. Let $K$ be a fixed closed convex set and let $C(t)$ be any curve that lies in $K$ for $-1 \leqq t \leqq 1$. Let $\alpha(t), t \in[-1,1]$, be a nonde- creasing function with at least one point where it is increasing. Then $U(K)$ is the domain of univalence of the class of all such functions of the form 


$$
f(z)=\int_{-1}^{1} \frac{d \alpha(t)}{z-C(t)} .
$$

The proof is the same as for Theorem 1.

As an illustration of Theorems 1 and 2 , let $K$ be the circular region $|z| \leqq 1$. Then the director curve is the circle $|z|=\sqrt{ }(2)$, and we obtain Cakalov's Theorem A and Corollary. If we let $K$ be the line segment, $-1 \leqq z \leqq 1$, then the director curve is the circle $|z|=1$, and we obtain Cakalov's Theorem B and Corollary.

As a further example, we may take for $K$ the set of four points $\{1,-1, i,-i\}$. Then the director curve is the union of four semicircles and the region of univalence for the family of functions

$$
f(z)=\frac{A_{1}}{z-1}+\frac{A_{2}}{z+1}+\frac{A_{3}}{z-i}+\frac{A_{4}}{z+i}, \quad A_{1}, A_{2}, A_{3}, A_{4}>0
$$

is the region shown shaded in Figure 1.

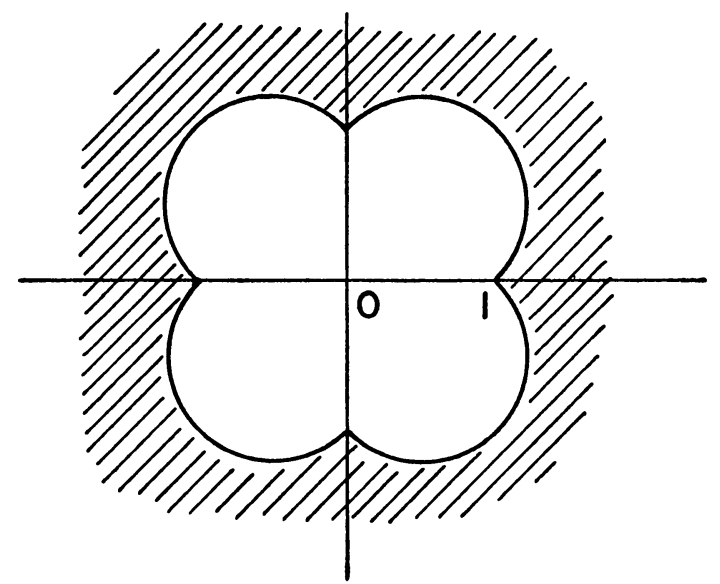

Figure 1

3. The ellipse. If we let the boundary of $K$ be an ellipse we get an interesting special case of Theorem 1 . It is well known $[1, \mathrm{p} .213]$, $[6$, p. 166], $[7$, p. 261], $[8$, p. 188] that the director curve for the ellipse $E$ :

$$
\frac{x^{2}}{a^{2}}+\frac{y^{2}}{b^{2}}=1
$$

is the circle 


$$
x^{2}+y^{2}=a^{2}+b^{2} .
$$

Now let $K$ be the closed region bounded by the ellipse $E: a^{2} x^{2}+y^{2}$ $=a^{2}$. Theorem 1 gives immediately:

THEOREM 3. The domain of univalence of the family of functions of the form (1) where each pole $a_{k}$ lies inside or on the ellipse $a^{2} x^{2}+y^{2}=a^{2}$ is the exterior of the circle $|z|=\sqrt{ }\left(1+a^{2}\right)$.

Observe that if $a=1$, we have a circle, and Theorem 3 gives Theorem A. If $a=0$, the ellipse of Theorem 3 collapses into a straight line segment and Theorem 3 gives Theorem B. Thus Theorem 3 fills a gap between Theorems $\mathrm{A}$ and $\mathrm{B}$ in a continuous manner.

Corollary. Let $\alpha(t), t \in[-1,1]$, be nondecreasing, with at least one point at which it is increasing. Then

$$
\int_{-1}^{1} \frac{d \alpha(t)}{z-(\cos t+i a \sin t)}
$$

is univalent in $|z|>\sqrt{ }\left(1+a^{2}\right)$.

4. Unbounded regions. Any unbounded region containing more than a quarter-plane would not have a director curve. The sector $0 \leqq \arg w \leqq \theta_{0}$ with $\theta_{0}<\pi / 2$ has a director curve which consists of the negative imaginary axis and the ray at the angle $\theta_{0}+\pi / 2$. If $K$ is a quarter-plane then the director set is not a curve but is another quarter-plane.

The half strip formed by the negative real axis, the segment $[0, i]$ and the line $y=1, x \leqq 0$ would have for its director curve the negative imaginary axis, the right half of the circle with center at $z=i / 2$ and radius $1 / 2$ and the imaginary axis above $z=i$. If we reduce the width of this half strip to zero we find that the domain of univalence of the class of functions of the form (1) where the $a_{k}$ are all negative real is the right half plane. This is a result of F. M. Reza [5] in his study of impedance functions.

The director curve for a parabola is the familiar directrix.

5. A generalization. We can allow the residues $A_{k}$ to be complex if we shrink the domain of univalence suitably. Let us extend Definition 1 as follows:

Definition 2. Let $K$ be a closed set of points. The director set of order $\delta$ of $K$ is the collection $D(\delta)$ of all points $P$ such that $K$ subtends an angle $\delta$ at $P$.

As before if $K$ is bounded and if $0<\delta \leqq \pi$ then $D(\delta)$ will be a simple 
closed curve which will divide the plane into two domains, one of which contains $K$ and the other we call $U(K, \delta)$.

Let $\mathcal{F}\left(K, \theta_{0}\right)$ be the family of functions

$$
f(z)=\sum_{k=1}^{n} \frac{A_{k}}{z-a_{k}}
$$

where all the poles lie in $K$ and $-\theta_{0} / 2 \leqq \arg A_{k} \leqq \theta_{0} / 2, k=1,2, \cdots, n$. Theorem 1 can now be extended to:

THEOREM 4. Let $K$ be a closed set for which $U\left(K, \pi / 2-\theta_{0}\right)$ is not empty. Then $U\left(K, \pi / 2-\theta_{0}\right)$ is the domain of univalence of the class of functions $\mathfrak{F}\left(K, \theta_{0}\right)$.

Proof. The proof is similar to that of Theorem 1.

6. Acknowledgment. I wish to thank Professor A. W. Goodman for suggesting this topic and his advice on the preparations of this paper.

\section{REFERENCES}

1. J. H. Shakleton Bailey, Elementary analytical conics, Oxford Univ. Press, London, 1936.

2. L. Cakalov, Sur une classe de fonctions analytiques univalentes, C. R. Acad. Sci. Paris 242 (1956), 437-439.

3. - Sur les domaines d' univalence de certaines classes de fonctions analytiques, Bŭlgar. Akad. Nauk. Izv. Mat. Inst. 4 (1960), no. 2, 43-55. (Bulgarian. Russian and French summaries)

4. M. Marden, The geometry of the zeros of a polynomial in a complex variable, Math. Surveys No. 3, Amer. Math. Soc., Providence, R. I., 1949.

5. F. M. Reza, On the schlicht behavior of certain impedance functions, IRE Trans. Circuit Theory, CT-9 (1962), 231-232.

6. G. Salmon, Conic sections, Longmans, Green and Co., London, 1879.

7. P. F. Smith and A. Gale, Analytic geometry, Ginn, New York, 1904.

8. R. Walker, Analytical geometry, Arnold, London, 1950.

UNIVERSITY OF KENTUCKY 